\title{
Hemorragia de intestino medio: Conceptos actuales. ¿Cápsula endoscópica, enteroscopia apoyada por dispositivos 0 arteriografía?
}

\author{
Gerardo Blanco-Velasco* \\ Servicio de Endoscopia, Hospital de Especialidades, Centro Médico Nacional Siglo XXI, Instituto Mexicano del Seguro Social, Ciudad de México, \\ México
}

\begin{abstract}
Resumen
La hemorragia de intestino delgado (HID) representa del 5 al 10\% de las hemorragias del tubo digestivo. La cápsula endoscópica está considerada el estándar de oro para el diagnóstico de las HID y la enteroscopia apoyada por dispositivos es el método de primera línea para el tratamiento de los mismos. La arteriografía es de utilidad en aquellos pacientes con inestabilidad hemodinámica. Durante IOS ESGE Days 2019 y la DDW 2019 se presentaron diversos trabajos relacionados con HID. De estos se realizó una revisión de los estudios más interesantes relacionados con endoscopia gastrointestinal, en los que se documentan adelantos en el diagnóstico y tratamiento de los mismos.
\end{abstract}

Palabras clave: Hemorragia de intestino delgado. Cápsula endoscópica y enteroscopia.

\section{Introducción}

La hemorragia de intestino delgado (HID) representa el 5 al $10 \%$ de todas las hemorragias del tubo digestivo. Las causas de hemorragia varían según la edad siendo la enfermedad inflamatoria intestinal, las lesiones de Dieulafoy, neoplasias, divertículos de Meckel y síndromes polipósicos las causas más comunes en menores de 40 años, mientras que las angioectasias, las lesiones de Dieulafoy, neoplasias y úlceras por antiinflamatorios no esteroideos (AINE) son más comunes en mayores de 40 años'. La cápsula endoscópica (CE) es considerada hoy en día como la primer línea de estudio en pacientes con sospecha de HID, mientras que la enteroscopia apoyada por dispositivos $(E A D)$ es el procedimiento de elección para realizar terapéutica en el intestino delgado². Debido a la alta eficiencia de la CE y la EAD, la arteriografía se ha reservado para aquellos pacientes con hemorragia masiva y/o datos de inestabilidad hemodinámica ${ }^{3}$.

\section{Trabajos realizados}

Durante los ESGE Days 2019, celebrados en Praga, República Checa del 4 al 6 de abril del 2019 se presentaron 10 trabajos relacionados con la hemorragia de intestino delgado, mientras que en la Semana de Enfermedades Digestivas realizada en San Diego, California, Estados Unidos del 18 al 21 de mayo del 2019, se presentaron 13 trabajos del mismo tema. A continuación se describirán algunos de los trabajos más importantes presentados.

\section{Correspondencia:}




\section{Objetivo}

El objetivo de esta revisión será analizar los trabajos de HID relacionados con la CE y EAD durante los ESGE Days 2019 y la DDW 2019.

\section{Desarrollo}

De los trabajos más importantes presentados en los ESGE Days 2019, el primer trabajo fue realizado por Davie, et al., quienes buscaron la localización más frecuente de las angioectasias en intestino delgado. Incluyeron 164 estudios de CE en que se reportaron la presencia de angioectasias. Observaron que de 554 angioectasias observadas, $435(78.52 \%)$ se encontraban en el primer tercio del tiempo de tránsito intestinal de CE, mientras que 227 (50\%) angioectasias se encontraban en el $10 \%$ inicial del tiempo de tránsito intestinal de la CE. Concluyen que cuando existe sospecha de angioectasias estas se deben buscar en el tercio proximal del intestino delgado e incluso pueden ser tratadas utilizando un endoscopio tradicional ${ }^{4}$.

Otro estudio interesante fue el presentado por Mussetto, et al. en Italia. La intención de este estudio fue valorar la utilización de la panendoscopia por CE en pacientes con melena y endoscopia superior negativa. Incluyeron a 12 pacientes. Identificaron la causa de la hemorragia en intestino delgado en 6 pacientes y en colon en 4 pacientes, identificando hallazgos significativos en intestino delgado y en colon en dos pacientes. Concluyen que la CE puede ser una herramienta eficiente para el diagnóstico en pacientes con melena previo a la realización de una colonoscopia ${ }^{5}$.

Gomes, et al. presentaron un trabajo realizado en Portugal, en el que valoraron el riesgo de resangrado en aquellos pacientes con una CE positiva seguida de una $E A D$ negativa. Incluyeron a 24 pacientes que tenían CE con los siguientes hallazgos: lesiones subepiteliales en 9 casos, hemorragia activa en 6 , lesiones inflamatorias en 5 , angioectasias en 2 y pólipos en 2 estudios. Se realizaron enteroscopias anterógradas en 15 pacientes, retrógradas en 8 y en un caso por ambas vías. No se observaron hallazgos en 13 pacientes y en 11 los hallazgos fueron no significativos. Se presentó resangrado en 11 pacientes (45.8\%), con una tasa de resangrado al mes, 1, 2, 3 y 4 años de $20.8 \%, 25.2 \%$, $35.9 \%, 50.6 \%$ y 62.9 , respectivamente. Todos los pacientes con resangrado tuvieron una segunda evaluación radiológica/endoscópica logrando u diagnóstico y tratamiento definitivo en solo 4 casos (2 lesiones vasculares y 2 tumores de intestino delgado).
Concluyen que los pacientes con enteroscopias falsas-negativas tienen una alta tasa de resangrado ${ }^{6}$.

Las angioectasias son la principal causa de hemorragia en el intestino delgado y generalmente sangran de forma intermitente. Por este motivo, Arieira, et al. estudiaron en Portugal cuáles son las características y posibles predictores de resangrado en estas lesiones. Incluyeron a 129 pacientes con HID por angioectasias. En el $68.2 \%$ de los casos la hemorragia fue oculta y evidente en $31.8 \%$. el $32.6 \%$ de los pacientes tuvo uno o más eventos de resangrado. Realizaron un análisis univariado observaron que los pacientes con resangrado son más viejos ( 74.2 vs $67.9 ; p=0.021$ ) y frecuentemente tienen patologías como enfermedad renal crónica (44.4\% vs $26.2 \% ; p<0.035)$ y cardiopatías $(51.9 \%$ vs $19.5 \%$; $<0.001)$. En cuanto a las características de las angioectasias, las de mayor tamaño (> $5 \mathrm{~mm})(69 \%$ vs $27.6 \% ; p<0.001)$ fueron factor de riesgo para resangrar. En el análisis multivariado, se observó que la razón de momios para resangrado de la cardiopatía fue de 3.3 (IC 95\% 1.3-8.6; $p=0.014$ ) y para el tamaño de las angioectasias de 4.9 (IC 95\% 2.1-11.4; $p<0.001$ ). Concluyen que la cardiopatía y las angioectasias mayores de $5 \mathrm{~mm}$ son factores de riesgo para resangrado. Este trabajo también fue presentado en la DDW 20197. México también estuvo presente en los ESGE Days 2019, presentamos un trabajo realizado en el Hospital de Especialidades del Centro Médico Nacional Siglo XXI. En este estudio evaluamos el rendimiento diagnóstico de la CE Pillcam SB3 y la CE OMOM en pacientes con hemorragia de intestino delgado. Se incluyeron a 20 pacientes con HID y último evento de hemorragia menor a 15 días a los que se les colocó de forma aleatoria ambas CE con un día de diferencia para no tener interferencia en la señal de las mismas. Se utilizó la clasificación de Saurin para dividir los hallazgos en lesiones P0, P1 y P2. Se logró visualización completa del intestino delgado en 18 SB3 y en 19 OMOM ( $p=0.548)$. La mediana del tiempo de tránsito intestinal fue de 355 con SB3 y de 240 con OMOM $(p=0.445)$. El tiempo de batería fue significativamente mayor con la SB3 (821 vs 703 minutos; $p<0.001$ ) y el tiempo de descarga de las imágenes fue menor con la OMOM (31 vs 117 minutos; $p<0.001$ ). Ambas CE presentaron una falla. Se identificó la causa de la hemorragia en 18 SB3 (90\%) y en 16 OMOM (80\%) ( $p=0.331)$. Se observaron lesiones P2 en 12 SB3 (60\%) y en 11 OMOM ( $p=0.749)$. Las lesiones $\mathrm{P} 1$ se identificaron en 3 pacientes con ambas cápsulas y las lesiones extraintestinales se encontraron en 3 pacientes con SB3 y en 2 con OMOM ( $p=0.633)$. Como conclusión no se 
encontraron diferencias significativas entre ambas CE para la identificación de la causa de hemorragia. Se observaron diferencias significativas en la duración de la batería y en el tiempo de descarga con ambos tipos de $\mathrm{CE}^{8}$.

En la DDW 2019 también se presentaron trabajos interesantes como el realizado por Wetwittayakhlang, et al. en Tailandia, que estudiaron los predictores de resangrado en pacientes con HID. Incluyeron a 134 pacientes con HID y CE de 2008 a 2018. Observaron que el $26.9 \%$ resangraron. La tasa de resangrado fue mayor en pacientes con CE positiva sin tratamiento específico (50\%) que en aquello con CE positiva y tratamiento específico (27.8\%) y CE negativa (19.7\%) $(p=0.019)$. La tasa de resangrado que ocurrieron en un periodo de 6, 12, 18 y 24 meses fue de $36.1 \%, 63.9 \%$, $77.8 \%$ y $91.7 \%$, respectivamente. El antecedente de HID previa (HR 3.35; IC 95\%, 1.17-9.58;p=0.023) y visualización incompleta del intestino delgado por CE (HR 7.12; IC 95\%, 2.16-23.50; $p=0.001$ ) fueron factores de riesgo para resangrado. Las angioectasias fueron las lesiones más comunes (47\%). Concluyen que el seguimiento de los pacientes con HID debe ser de al menos 2 años ${ }^{9}$.

Otro estudio interesante fue el de Shetty, et al, de Canadá que estudio la tasa de resangrado en pacientes con HID por lesiones vasculares y tratamiento por EAD. Incluyó a 23 artículos con 791 pacientes y observó resangrado en $37.4 \%{ }^{10}$.

Considerando los estudios comentados podemos concluir que las angioectasias son la principal causa de HID, que la mayoría de ellas se encuentran en el primer tercio del intestino delgado y que tienen una alta tasa de resangrado a pesar de ser tratadas con EAD. Que la CE tiene mayor utilidad para el diagnóstico de HID en pacientes con melena y endoscopia superior negativa; y que la tanto la CE Pillcam SB3 como la CE OMOM son efectivas para el diagnóstico de HID.

\section{Conflicto de intereses}

No existen conflictos de interés. Sin patrocinio de la industria.

\section{Bibliografía}

1. Gerson LB, Fidler JL, Cave DR, et al. ACG Clinical Guideline: Diagnosis and Management of Small Bowel Bleeding. Am J Gastroenterol 2015; 110:1265-1287.

2. Chetcuti Zammit S, Sidhu R. Small bowel bleeding: cause and the role of endoscopy and medical therapy. Curr Opin Gastroenterol 2018;34:165-174.

3. Gerson LB. Small Bowel Bleeding: Updated Algorithm and Outcomes. Gastrointest Endosc Clin N Am 2017;27:171-180.

4. Davie M, Yung D, Plevris J, et al. Mapping the distribution of small bowel angioectasias. Endoscopy 2019;51:S10.

5. Mussetto A, Arena R, Triossi O. Pan-enteric capsule in patients with melena and a negative upper endoscopy: A pilot study. Endoscopy 2019; $51:$ S30.

6. Gomes AC, Pinho R, Rodrigues A, et al. high reebleding rate in patients evaluated for obscure gastrointestinal bleeding after a false-negative deep enteroscopy. Endoscopy 2019;51:S31-S32.

7. Arieira C, Magalhaes, De Castro FD, et al. Small bowel angioectasias rebleeding and identification of higher risk patients. Endoscopy 2019;51:S8.

8. Blanco-Velasco G, Solorzano-Pineda OM, Zamarripa-Mottu, et al. Comparison of the diagnostic yield of "Pillcam SB3" and "OMOM" capsule endoscopy in small bowel bleeding. Endoscopy 2019;51:S30.

9. Wetwittayakhlang $\mathrm{P}$, Wonglhow J, Netinatsunton $\mathrm{N}$, et al. Long-term re-bleeding and its predictors after capsule endoscopy in patients with obscure gastrointestinal bleeding.

10. Shetty $A$, Tofighi $H$, Hansen $T$, et al. Long term rebleeding outcomes after endoscópic therapy with balloon assisted enteroscopy for small bowel vascular lesions: a meta-analysis. 